\title{
Growth Hormone Deficiency in Adults: Impact of Replacement Therapy
}

\author{
J.S. Christiansen and J.O.L. Jørgensen \\ Department of Endocrinology, Aarhus University Hospital, Aarhus C, Denmark
}

Key words: GH, adults, growth

\section{Introduction}

In his monograph "the pituitary gland" from 1912 Harvey Cushing was the first to introduce the expression: the hormone of growth-by that in effect giving the hormone the name of growth hormone. It had for some years been suspected that there from the pituitary gland was released a hormone with action on growth and body composition, but unfortunately growth hormone became the accepted name implying that the hormone only processed one important action: i.e. the action on skeletal growth.

In his beautiful review from 1962 (1) Raben, however, described almost all the actions of growth hormone that we know today. He also suggested that growth hormone was necessary for the normal maintenance of adults tissues and he described a treatment trial in one adult patient suffering from hypopituitarism who-in addition to traditional replacement-was treated with growth hormone with favourable results (1).

For a long time no further reports on adult GH replacement was offered. However, in 1986 beneficial effects on cognitive functions in five

Correspondence: Dr. J. S. Christiansen, Department of Endocrinology, Aarhus University Hospital, N>rrebrogade 44, 8000 Aarhus C, Denmark adults growth hormone deficient (GHD) patients receiving $8 \mathrm{IU}$ GH i.m. three times a week for eight weeks was reported (2). In 1987 increased fat free mass and reduced fat mass was seen in six female adult GHD patients receiving exogenous growth hormone for two weeks (3).

With the advent of biosynthetic human growth hormone the problem of limited growth hormone available was solved and clinical trials on extended use of the hormone could commence.

\section{The First Controlled Trials}

The two first placebo-controlled trials on the effect of GH replacement therapy in adult GHD patient was published in $1989(4,5)$. The two studies were quite different in design, patient material, methods employed, and dose given. The Danish study reported on a cross-over trial with a four month treatment period and a four month wash-out period in between, while the English study had a parallel design of six month duration. All the patients recruited for the Danish study had childhood onset GHD while 22 of the 24 patients in the English study had growth hormone deficiency acquired in adult age $(4,5)$. The actual age of the patients were also different with a mean age of 24 in the Danish study and 39 years in the English study and the dose of $\mathrm{GH}$ used differed from $2 \mathrm{IU} / \mathrm{m}^{2}$ subcutaneous per day in Denmark to $2.8 \mathrm{IU} / \mathrm{m}^{2}$ daily in En- 
gland.

Despite these marked differences in patient material, design, and methods the results obtained are strikingly similar. Both studies found a significant reduction in fat mass and an increase in the fat free mass. The muscle fat ratio was found to be significantly decreased compared to normal subjects and showed a normalization. Improved muscle strength exercise, capacity, cardiac contractility, and renal function was also reported $(4,5)$.

\section{Effects of Adult GH Replacement}

It is increasingly evident that one of the major actions of growth hormone in the adult body after cessation of skeletal growth is to act as a stress hormone. Fasting causes a sharp rise in GH secretion whereby the pronounced lipolytic effect of the hormone facilitates the mobilisation of energy from adipose tissue-and thereby indirectly has a protein saving effect. The administration of glucose suppresses while ami- no acid stimulates GH secretion. The latter presumably results an increased protein synthesis. Thus GH is presumed to be of major importance for the maintenance of total muscle mass-and in particular to act as an anticatabolic hormone during caloric restriction.

The importance of growth hormone for normal fuel utilisation was recently demonstrated in six young GHD adults on ongoing replacement therapy (6). These patients participated in a trial in which they in random order received $\mathrm{GH}$ or saline infusion for 24 hours during monitoring of intermediate metabolism. The data were compared to data obtained in normal control subjects. In this way the effect of one day GH deprivation was analyzed. On the day off $\mathrm{GH}$-substitution a significant increase in protein energy expenditure and a corresponding decrease in lipid oxidation was seen in these patients compared to the normal values obtained during ongoing GH replacement (Fig. 1). Thus it was demonstrated that during GH deficiency the body are more prone to utilize protein as energy source

\section{Six GHD adults \\ on ongoing \\ $\mathrm{GH}$ replacement \\ Mean age 22 yrs \\ Mean BMI 21.7 \\ $\mathrm{kg} / \mathrm{m}$}
- RECEIVING EITHER GH OR SALINE TIV FOR ONE DAY

- compared to NORMAL CONTROLS $(n=7)$
Protein EE

$(\mathrm{mg} / \mathrm{kg} \cdot \mathrm{min})$
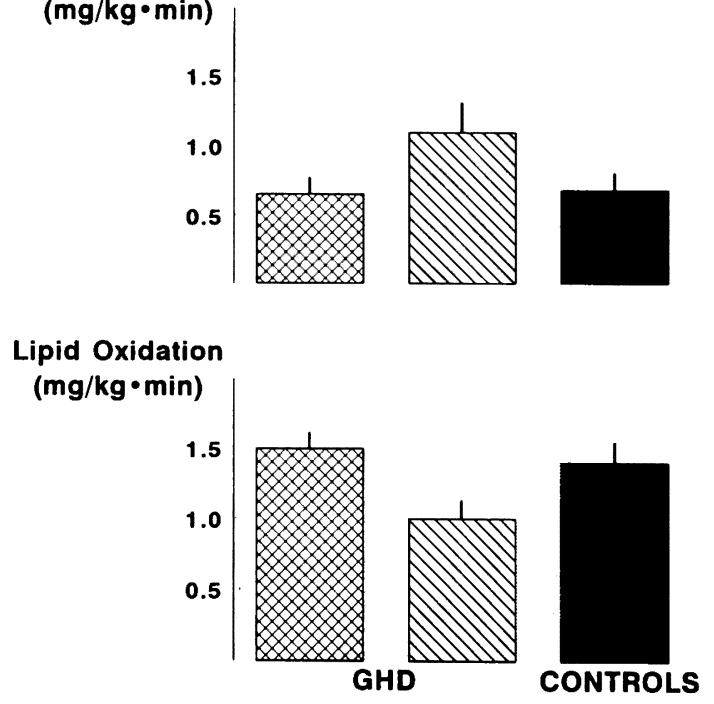

Fig. 1 Metabolic aspects of GHD. Effect of one day GH deprivation on protein and lipid metabolism in GHD patients on ongoing $\mathrm{GH}$ substitution therapy (6). 
than lipid-which beautifully explains the accumulation of body fat and reduction in muscle mass in adult GHD patients (6).

Numerous studies have subsequently confirmed and extended the original observations from 1989. It was soon demonstrated that the highly significant reduction in fat mass showed an uneven regional distribution. Thus, the most pronounced reduction in fat mass was seen in visceral or intraabdominal fat (7).

The symptomatology of GHD in adults often includes complaints of lack of muscle strength and early fatigue. Isometric muscle strength as measured in the quadriceps is significantly reduced in GHD patients (8) and numerous studies have reported on increased muscle strength following replacement-although no controlled trials so far have been able to show treatment effect of statistical significance. All data taken together, however, there can be no doubt that $\mathrm{GH}$ replacement improves muscle strength.

In several studies exercise capacity has been measured by means of an electromechanical bicycle with or without concurrent analysis of maximal oxygen uptake. In untreated GHD patients both exercise capacity and maximal oxygen uptake are considerably reduced by approximately $25 \%$ compared to normal individuals. Placebo-controlled trials have shown a distinct increase in exercise capacity after 4-6 month of daily replacement $(4,8)$.

A list of parameters - all of which have been proven to be subnormal in the untreated adult GHD - and where improvement have been proven in controlled clinical trials-are given in Table 1 together with selected references.

We have now experience with uninterrupted replacement therapy for more than six years. At the five years assessment in seven patients the muscle and fat volume were fully comparable to the values obtained in matched control subjects (Fig. 2).

\section{Clinical Management of Adult GHD}

\section{Diagnosis}

The criteria for the diagnosis of GHD in adults currently used in our country is given in Table 2. It will be seen that very strict criteria for this indication is presently required.

\section{Dose}

Dosing of growth hormone is highly individual-just like insulin. Recommended starting dose is 0.5 IU subcutaneously in the evening with a gradual-and slow-increased in dose at 4 week intervals up to an approximate dose of 1.0-1.5 $\mathrm{IU} / \mathrm{m}^{2} /$ day. After the age of 40 a gradual decline in the replacement dose is likely.

\section{Side effects}

The only reported side effects all relates to the acute fluid retention seen after commencement of $\mathrm{GH}$ replacement. It is important to realise that this increase in body water and extracelluar fluid volume represents a normalization of these parameters and therefore academically could be considered effects rather than side ef-

Table 1 Effects of GH replacement therapy in adult GHD patients

The following parameters-all known to be abnormal in the adult GHD syndrome-have proven to be completely or partly normalized by GH in controlled trials:

Muscle/fat ratio
Visceral obesity
Fluid homeostasis
Renal function
Exercise capacity
Sweat secretion
Muscle strength
Lipid profile
Cardiac contractility
Quality of life


Muscle

$\mathrm{cm}^{2} / 10 \mathrm{~mm}$ slice

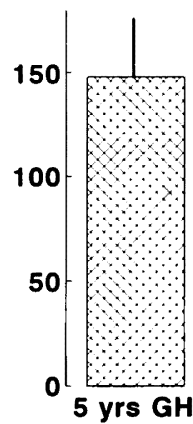

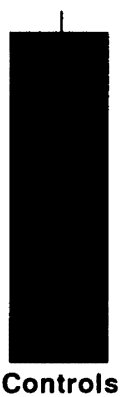

FAT

$\mathrm{cm}^{2} / 10 \mathrm{~mm}$ slice

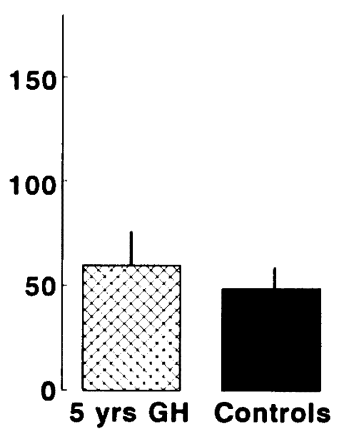

Fig. 2 Mid femoral CT-scan. Muscle and fat volume at the thigh in 7 GHD adults after 5 years of GH replacement as compared to matched control subjects.

Table 2 Criteria for the diagnosis of GHD in adults

\begin{tabular}{ll}
\hline Either - & GHD diagnosed in childhood \\
& and \\
- & insufficient increase in serum GH after a new GH stimulation test \\
\hline Or $\quad-$ & insufficient increase in serum GH after a GH stimulation test in \\
& patients with known pituitary pathology \\
& and \\
- & documented failure of a least one further pituitary hormone (folli- \\
& cle-stimulating hormone, luteinising hormone, adrenocorticotrophic \\
& hormone or thyroid-stimulating hormone)
\end{tabular}

fects. However, in order for the patients not to experience this rapid change in body fluid homeostasis as a discomfort it is advisable to start replacement of GH gradually with a careful and slow increase in dose as indicated above.

So far no indications of hypertension, cardiac hypertrophy, deterioration in glucose homeostasis or tumour recurrence have been reported.

\section{Conclusion}

The lack or cessation of GH secretion from the pituitary gland results in an abnormal metabolic condition in which e.g. fasting and stress are characterized by relatively increased protein and reduced lipid metabolism. GHD is not characterized by symptoms that are as quickly recognisable and conspicuous as those seen in failures of other hormonal systems, e.g. insulin, cortisol, thyroid or sex hormones.

Long lasting untreated GHD results in a number of symptoms of organic dysfunction. Individually the symptoms may be of less importance and as times goes by they may be overshadowed by-or seen as-symptoms of incomplete replacement of the other hormonal axes in the completely or partly panhypopituitary condition with which GHD is usually combined. Taken together, however, all these symptoms 
have distinct consequences and it has now been clearly shown that untreated GHD patients usually are not capable of living a normal life. Furthermore it must be considered as proven that GH replacement by a large number of patients is perceived as being associated with an improvement in their quality of life.

It is our firm belief that $\mathrm{GH}$ replacement therapy should be considered in all adult GHD patients. In the next decades it is, however, mandatory that we centralize the treatment of these patients and collect data in order to monitor possible (albeit unlikely) side effects.

\section{References}

1. Raben MS. Growth hormone. N Eng J Med 1962; 266: 82-6.

2. Almqvist O, Thoren M, Saaf M, Eriksson O. Effect of growth hormone substitution on mental performance in adults with growth hormone deficiency. A pilot study. Psychoneuroendocrinology 1986; 11: 347-52.

3. Crist DM, Peake GT, Markonnon LT, Sibbitt WL, Kraner JC. Exogenous growth hormone treatment alters body composition and increases natural killer cell activity in women with impaired endogenous growth hormone secretion. Metabolism 1987; 36: 1115-7.

4. Jørgensen JOL, Thuesen L, Ingemann-Hansen T,
Pedersen SA, Jørgensen J, Skakkebæk NE, et al. Beneficial effects of growth hormone treatment in GH deficient adults. Lancet 1989; 1: 1221-5.

5. Salomon F, Cuneo RC, Hesp R, Sonksen PH. The effects of treatment with recombinant human growth hormone on body composition and metabolism in adults with growth hormone deficiency. N Eng J Med 1989; 321 (26): 1797803.

6. Jørgensen JOL, Møller J, Alberti KGMM, Schmitz $\mathrm{O}$, Christiansen JS, Ørskov H, et al. Marked effects of sustained low growth hormone (GH) levels on day to day fuel metabolism: Studies in GH deficient patients and healthy untreated subjects. J Clin Endocrinol Metab 1993; 77 (6): 1589-96.

7. Bengtsson BAa, Eden S, Lonn L, Kvist H, Stokland $\mathrm{A}$, Lindstedt $\mathrm{G}$, et al. Treatment of adults with growth hormone (GH) deficiency with recombinant human GH. J Clin Endocrinol Metab 1993; 76 (2): 309-17.

8. Cueno RC, Salomon F, Wiles CM, Hesp R, Sonksen $\mathrm{PH}$. Growth hormone treatment in growth hormone deficient adults. I effects on muscle mass and strength. J Applied Physiol 1991; 70 (2), 688-94

9. Pedersen SA, Welling K, Michaelsen KF, Jørgensen JOL, Christiansen JS. Reduced sweating in adults with growth hormone deficiency. Lancet 1989; 2 : 681-2. 